\section{Steroid Resistant Nephrotic Syndrome with Clumsy Gait Associated With INF2 Mutation}

There has been significant improvement in our understanding of steroid resistant nephrotic syndrome (SRNS) with identification of multiple newer genes that are involved in regulating podocyte protein and maintaining podocyte architecture. Mutations in these genes have been linked to various forms of SRNS [1]. We hereby describe a child with SRNS whose associated neurological problem gave us a clue to the underlying etiology and genetic analysis provided the most likely link between his SRNS and neurological manifestations.

A 3-year-old boy born out of a non-consanguineous marriage presented with generalized swelling of body and decreased urine output. Blood and urine investigations confirmed the diagnosis of nephrotic syndrome. Remission was not achieved despite six weeks of full dose ( $2 \mathrm{mg} / \mathrm{kg}$ bodyweight) prednisolone and child was classified as SRNS. Renal biopsy revealed focal segmental glomerulosclerosis (FSGS) histopathology and tacrolimus therapy was added. Proteinuria failed to respond to tacrolimus as well as to subsequent addition of rituximab. During this period the child's gait was noticed to be clumsy. Neurological examination was unremarkable apart from mild bilaterally diminished ankle jerks. Nerve conduction velocity study was consistent with bilateral motor axonal neuropathy in lower limbs suggestive of Charcot Marie Tooth disease (CMTD). Next Generation Sequencing (NGS) and analysis of the exome data for any copy number variation revealed a heterozygous deletion (chr14:105181575-105196577) involving the INF2 gene, which was extending to the proximal part of an adjoining gene ADSSL1. ADSSL1 gene is implicated for distal myopathy-5 but as this is inherited in autosomal recessive fashion; the heterozygous deletion in our child was deemed insignificant. On the other hand, INF2 mutation, which is inherited in an autosomal dominant mode, has been linked with FSGS coexisting with CMTD. Financial constraint precluded further confirming the CNV by cytogenetic microarray or to look for inheritance.

Large deletions involving the region described in our patient has been reported as pathogenic in genetic databases but phenotype similar to ours were not reported. Considering the above facts, the CNV found in our patient was classified as VUS (Variation of unknown significance). NGS can sometimes fail to detect deletions or duplication beyond few nucleotides and even in our case initial report was negative. In view of high suspicion, re-analysis of data was undertaken wherein with the aid of newer bio-informatic tools, the large deletion was identified. Currently the child is on regular albumin / frusemide infusion through a portacath along with anti-proteinuric agents like angiotensin converting enzyme inhibitor (ACEi). Although the association of CMTD with FSGS has been known for quite some time, the molecular pathogenesis linking the two has only been recently described with reports of INF2 mutations in up to $75 \%$ of cohorts with this combination $[2,3]$. The INF2 protein is a member of the formin family of actin-regulatory proteins with an $\mathrm{N}$-terminal Diaphenous Inhibitory Domain (DID) formin homology 1 and 2 domains and a C-terminal WASP Homology 2 domain, which has the hallmarks of the diaphanous autoregulatory domain (DAD) similar to other formins [4]. The CNV although currently classified as VUS in our child is particularly important as unlike all other previously reported mutations which were in the DID region, our case had it in the DAD region. Although interaction between the DID and DAD has been reported to be crucial in regulating INF2 depolymerization [5], clinical cases with mutations in DAD region have not been described earlier. INF 2 is strongly expressed in Schwann cell cytoplasm and interacts with myelin and lymphocyte protein (MAL2) and with GTP binding protein CDC42. These are essential for myelination and maintaining myelin structural integrity explaining the pathogenesis of CMTD. Proteinuria probably results from disruption in cytoskeletal dynamics due to defect in actin polymerization depolymerization balance secondary to INF2 mutation [2]. INF2 mutation has also been reported to result in isolated autosomal dominant FSGS without CMTD and it is postulated that the relative positions of the deletions results in different clinical manifestation $[2,3]$.

Our case not only highlights the interesting association of CMTD with FSGS, it also underscores the importance of NGS and the application of newer bio-informatic tools in the current genetic era. Among children with SRNS, recent guidelines strongly advocate the use of NGS for exome sequencing [1]. As in our child, additional clinical clues such as involvement of other systems are important and failure of a child with SRNS to respond to multiple immunosuppressant like calcineurin inhibitors (tacrolimus) and rituximab further augments the need to extensively search for an underlying genetic etiology.

RAJIV Sinha $^{1,2 *}$, RANIT MAITI ${ }^{2}$, Debaditya Das ${ }^{2}$ AND KaUSIK MandaL ${ }^{3}$

From ${ }^{1}$ Institute of Child Health; and ${ }^{2}$ Apollo Gleneagles Hospital, Kolkata, West Bengal; and ${ }^{3}$ Department of Medical Genetics, Sanjay Gandhi Post Graduate Institute, Lucknow, Uttar Pradesh; India.

\section{REFERENCES}

*rajivsinha_in@yahoo.com

1. Bensimhon AR, Williams AE, Gbadegesin RA. Treatment of steroid-resistant nephrotic syndrome in the genomic era. Pediatr Nephrol. 2019;34:2279-93.

2. Boyer O, Nevo F, Plaisier E, Funalot B, Gribouval O, Benoit G, et al. INF2 mutations in Charcot-Marie-Tooth disease with glomerulopathy. NEngl J Med. 2011;365:2377-88.

3. Caridi G, Lugani F, Dagnino M, Gigante M, Iolascon A, Falco M, et al. Novel INF2 mutations in an Italian cohort of patients with focal segmental glomerulosclerosis, renal failure and Charcot-Marie-Tooth neuropathy. Nephrol Dial Transplant. 2014;29:iv80-6.

4. Higgs HN, Peterson KJ. Phylogenetic analysis of the formin homology 2 domain. Mol Biol Cell. 2005;16:1-13.

5. Chhabra ES, Ramabhadran V, Gerber SA, Higgs HN. INF2 is an endoplasmic reticulum-associated formin protein. J Cell Sci. 2009; 122:1430-40. 